\title{
Design And Implementation Of Skeletal Prosthetic Arm For Paralyzed People
}

\author{
Dr.T.S.Udhayasuriya ${ }^{1}$, N.Jayasree ${ }^{2}$, K.Nageshwari ${ }^{3}$, V.Nivetha ${ }^{4}$, E.Priyadharshini ${ }^{5}$ \\ ${ }^{1}$ Head of the Department, ${ }^{2,3,4,5}$ Students \\ Department of Biomedical Engineering ${ }^{1,2,3,4,5}$ \\ Adhiyamaan College of Engineering, Hosur - 635109, India ${ }^{1,2,3,4,5}$ \\ Email: jayasreenagarathinam@gmail.com, ${ }^{2}$ nivevetri26@gmail.com ${ }^{4}$
}

\begin{abstract}
Paralysis is a impairment of voluntary muscular movement due to structural abnormalities of worried tissue. In gift facts, because of nutrient deficiency and lots of various predominant causes, Epilepsy is extensively noticed anywhere within the global. Such affected/paralyzed human beings in particular with hand disability revel in chargeable for being counting on others for their each day sports sports. So this paper proposes the format of a skeletal prosthetic arm which aids the affected man or woman to carry out his/her sports independently. Autonomy of the tool is completed thru the use of a particular manage tool, equipment assembly and stepper automobiles with the speech enter. The meant use of this era is in the scientific subject as a prosthesis, no matter the fact that the hand will also be adapted to paintings on distinctive robot structures as bendygripper
\end{abstract}

Index Terms- Bluetooth, Gear motor, Android app.

\section{INTRODUCTION}

A robotic can be described as an electromechanical device, that is capable of sensing it surrounding and taking its selection (command). In well known, robotic have to be able to pass (with the aid of using mechanical movement), it is able to moreover capable of revel in and it must be take decision (with the aid of voice manage). A robotic arm is a robotic manipulator which could carry out similar capabilities to a human arm. Robotics arm is vital feature of business application. most robotics arm perform the assignment together with welding, trimming, picking, setting and painting and plenty of others., moreover the most crucial advantage of these fingers is that it could work in hazardous areas and additionally in the regions which can't be accessed via human few versions are Keypad controlled, Voice manipulate, Gesture control, and so forth. But, most of the industrial robots are although programmed using the standard teaching method which continues to be a tedious and timeingesting mission that requires technical facts. Therefore, there may be a need for brand spanking new and simpler strategies for programming the robots. The high intention of this mission is the platform began with motion as fast due to the fact the voice command obtained through the usage of operator. The intention of this paper is to increase methodologies that help clients to control and software a robot, with a immoderate-degree of abstraction from the robotic particular language i.e. to simplify the robot programming.

\subsection{Existing System}

Bio mechatronics design of a Robotic arm for paralyzed people which are based on the remote control. Design and evalution of a soft and wearable Robotic glove for disabled hand which is based on the hand gestures.In this method, only remote controlled prosthetic hand is done. High cost and real time implementation is difficult.

\subsection{Proposed System}

The aim considerations taken during the design process were to design a hand that resembles the human hand, the size should be similar, light and not too bulky and the overall cost should be low. Our design can be trained using voice commands and can perform certain actions like picking up and releasing an object. Therefore we have shown that synchronization or real time operation of a speech recognition controller and a DC motor controller is possible. The prosthetic hand and the driving force for hand movements were obtained using miniature high-speed DC motor with a gearbox.

\section{LITERATURE SURVEY}

A layout of a operating robot arm which takes in voice/speech signals the usage of a speech processing unit and microcontroller. They pre-coded the necessary moves of the automobiles to carry out one-of-a-kind tasks. A speech popularity module became trained for recognizing the inputs like 'move forward' and many others and carried out in actual time. ${ }^{[1]}$

The excessive performance in electricity and precision of a humanoid arm/hand with the aid of using three actuation standards Electromagnetic joint lock mechanism, twisting actuation and disbursed actuation. We got taken thought from the aforementioned works and designed a prosthetic arm which could do most of the typical tasks required by means of an amputee. ${ }^{[2]}$

The layout of a 3 fingered robot pinnacle limb that might take human voice instructions as 


\section{Available online at www.ijrat.org}

inputs. They configured the elbow joint. Ventured a technique to simplify robot programming. Their purpose became to make the complicated technical languages used for robotic programming greater intuitive, less complicated, and faster to recognize. ${ }^{[3]}$

\section{SYSTEM IMPLEMENTATION}

\subsection{Block Diagram}

The Block diagram for layout and implementation of skeletal prosthetic arm for paralyzed human beings is shown in given under.

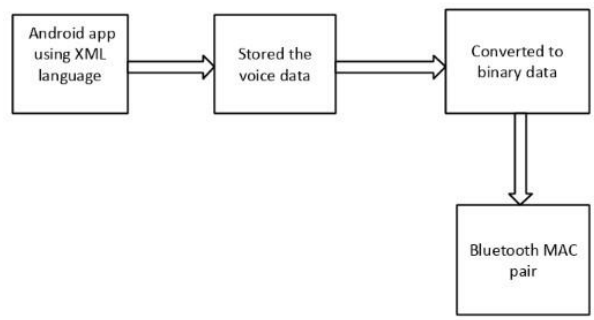

Fig 1 Transmitter block

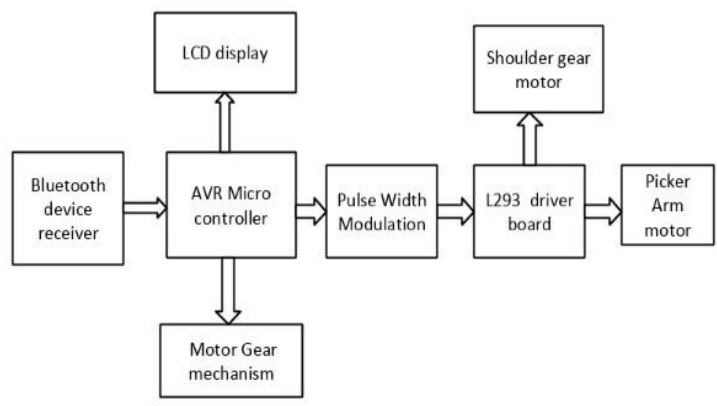

Fig 2 Receiver Block

\subsubsection{Block Diagram Description}

The movement of the robotic arm is managed using voice instructions i.e., (I) tools container, (ii) gripper rely upon the voice command given. The voice signal is captured the use of inbuilt microphone and is transmitted over a USB cable to an external on line server, in which it is converted into speech layout and essential instructions and speech is the top chassis has an microcontroller. two DC cars are used to govern the motion of the robotic arm. Another two DC vehicles are used to govern the motion of the robot arm and the robot arms. Motor driving force IC is used to manipulate the movement of DC motor. A single motor motive force (L293D) IC can control DC cars. A widespread electricity source cable is connected to microcontroller which interfaces with Bit Voice Server to transfer the speech signals. relying upon the commands given the robot performs moves consequently. Movement of the robot hand and gripper are independent of each other's moves. Side view with the robotic arms open is front view of robot voice. The top deck of the robotic has a microcontroller due that's used for taking the voice commands, synthesizing speech, and also up and down the instructions to the controller. Forwarding instructions from one microcontroller to some other is completed by way of the use of a pin to pin connection. If a particular pin is made high then that value is forwarded microcontroller. Then the code in Uno checks for the energetic pin and executes the precise function.

\section{CIRCUIT DIAGRAM}

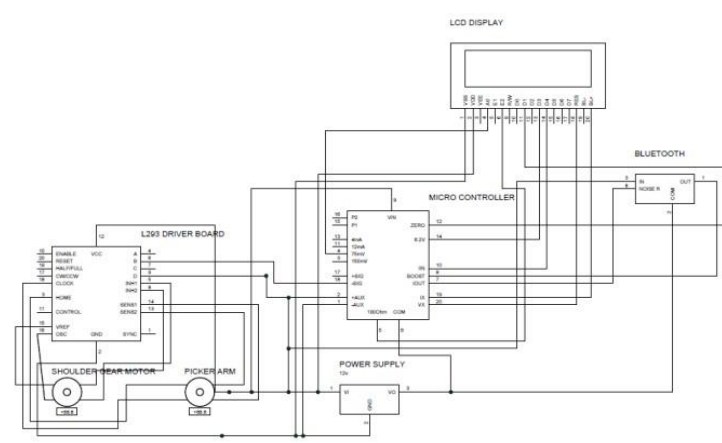

Fig 3 Circuit Diagram

\subsection{Circuit Description}

Exchange in rotation path of the DC Motor, generates Jaw Open \& close motion. The DC motor may be results easily be managed with the assist of DPDT switch (manual mode) or with the assist of any microcontroller along side aspect L293D Motor purpose pressure module. it could be used in numerous 'pick out and vicinity' type of robots. it really works on DC Motor (nine to $12 \mathrm{~V}$ DC). change in rotation course of the DC Motor, generates Jaw Open \& close to movement. The DC motor can be effects be managed with the assist of DPDT transfer (guide mode) or with the help of any microcontroller along side L293D Motor driver module. offers a similarly capability for your robots via including a totally purposeful robotic gripper to them. right here 1293 and 1298 performs comparable operations. This tool is a monolithic incorporated excessive voltage, excessive modern 4 channel motive pressure designed to really receive well known DTL or TTL commonplace experience ranges and pressure inductive masses (collectively with relays solenoids, DC and stepping cars) and switching energy transistors. every pair of channels is prepared with an permit input. A separate supply input is furnished for the coolest judgment, permitting operation at a decrease voltage and inner clamp diodes are blanketed. This tool is suitable to be used in switching applications at frequencies as much as $5 \mathrm{kHz}$. The Motor guard is able to force 2 servo automobiles, and has eight half of-bridge outputs for 2 gear cars or four 


\section{Available online at www.ijrat.org}

complete H-bridge motor outputs or 8 1/2-bridge drivers, or a combination. The servo automobiles use the $+5 \mathrm{~V}$ of the microcontroller board. The voltage regulator on the microcontroller board can also want to get warm. To keep away from this, the greater latest Motor Shields have connection elements for a separate $+5 \mathrm{~V}$.

\section{RESULT}

The tool turned into tested by using a healthy young grownup for initial test, in which the actuators will provide sluggish and secure motions. A controller with ON/OFF voice reputation is used to manipulate the robotic motions. Voice reputation are also prepared for the user to pick out the type of exercising for rehabilitation. In all 4 sorts of easy sporting events, the consumer has his/her arm straight pointing to the floor, and then bending the elbow assist via the robot arm either to, shoulder up, shoulder down, open, close, rotate $180 \mathrm{deg}$ according to the chosen form of workout.

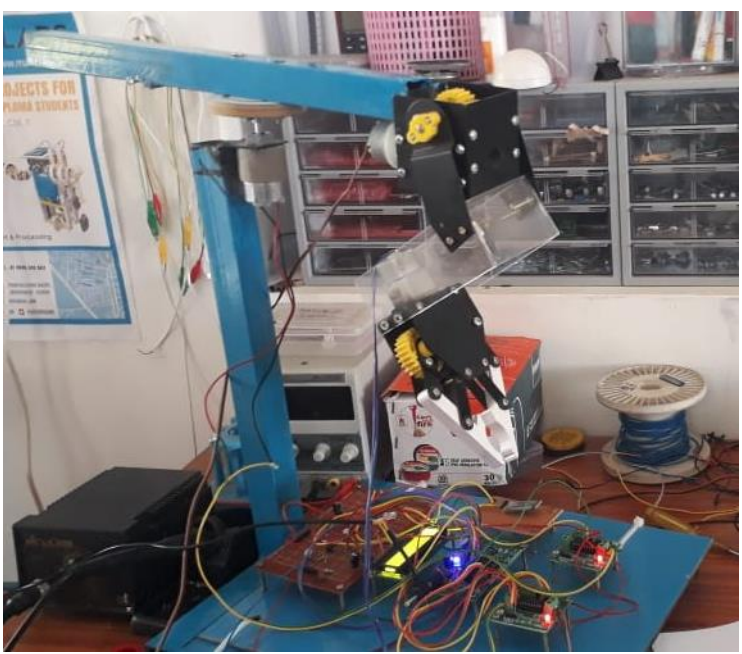

Fig 4 Output

\section{CONCLUSION}

In this challenge, we designed and applied a voice-managed prosthetic hand. Our undertaking involved each mechanical and electronic controller designs of the prosthetic hand. We have been able to function the prototype of the designed prosthetic hand at laboratory situations for simple choose up and launch responsibilities correctly. In our layout of the prosthetic hand, the use of pressure for hand motions turn out to be acquired using miniature high speed DC vehicles with a gearbox. each arm most effective maximum important joint is driven with the beneficial resource of the DC motor; the final joints are moved thru a unique mechanism regarding movement transmission bars. After layout and finding out of the prosthetic hand within the software surroundings, we synthetic a prototype of the prosthetic hand the usage of speedy prototyping techniques.

\section{FUTURE SCOPE}

Robot palms has a extensive scope of improvement. In the close to future the arms might be able to carry out each task as humans and in much better way. It is able to be a actual boon for handicapped people, who're paralyzed or lost their arms in some twist of fate. The arm can be trained to concentrate to the command from a human and carry out that mission. A unique gesture managed machine is also feasible. Wearable devices may be used to send the command and control the actions of the arm mind laptop Interface (BCI) is an immerging subject of research. BCI can be used to gather signals from the human brain and manipulate the arm. The machine can work in the same way as human arm. a person who can also have misplaced his hand in any twist of fate can resume his lifestyles like preceding with the aid of such artificial arms. robot fingers are versatile and feature sizeable methods of implementations.

\section{ACKNOWLEDGMENTS}

We thankful to Dr.T.S.UDHAYA SURIYA, M.E., Ph.D., Head of the Department of Biomedical Engineering, Adhiyamaan College of Engineering, for her most valuable guidance, advice and encouragement in all stages of our project

We would like to extend our gratitude to Dr.T.S.UDHAYA SURIYA, M.E., Ph.D., Head of the Department of Biomedical Engineering, Adhiyamaan College of Engineering, for her valuable guidance and support to complete our project successfully.

\section{REFERENCES}

[1] D. Osswald, J. Martin, C. Burghart, R. Mikut, H. Worn, G. Bretthauer, "INTEGRATING A FLEXIBLE ANTHROPOMORPHIC ROBOTIC HAND INTO THE CONTROL SYSTEM OF A HUMANOID ROBOT", Robotics and Autonomous Systems, Vol. 48, pp. 213-221.

[2] [2] J.K. Salisbury, M.T. Mason, ROBOT HANDS AND THE MECHANISM OF MAIPULATION, Cambridge, MIT Press.

[3] Otto Bock Healthcare, Minneapolis, MN, USA, http://www.ottobockus.com.

[4] Touch EMAS, Ltd., Edinburgh, UK, http://www.touchbionics.com.

[5] P.J. Kyberd, O.E. Holland, P.H. Chappel, S. Smith, R. Tregdigo, P.J. Bagwell, M. Snaith, "Marcus: A TWO DEGREE OF FREEDOM HAND PROSTHESIS WITH HIERARCHICAL GRIP CONTROL", IEEE Trans. Rehabil. Eng., Vol. 3, pp. 
International Journal of Research in Advent Technology, Vol.7, No.3, March 2019 E-ISSN: 2321-9637

Available online at www.ijrat.org

[6] P.A. O'Neill, E.L. Morin, R.N. Scott, "MYOELECTRIC SIGNAL

CHARACTERISTICS FROM MUSCLES

IN RESIDUAL UPPER LIMBS," IEEE

Trans. Rehabil. Eng., Vol. 2, pp. 266-270 